\title{
Differentiation of two subtypes of intrahepatic cholangiocarcinoma: imaging approach
}

\author{
Kengo Yoshimitsu ${ }^{1}$ (D)
}

Received: 16 February 2019 /Revised: 23 February 2019 / Accepted: 7 March 2019 / Published online: 26 March 2019

(C) European Society of Radiology 2019

\section{Key Points}

- Mass-forming intrahepatic cholangiocarcinoma can be divided into two subgroups, namley, perihilar and peripheral types.

- These two significantly differ in clinico-biological behaviors, and in patients' prognosis as well.

- This differentiation may be achieved solely by contrast-enhanced MR imaging findings.

An important and recent concept about the development of cholangiocarcinoma has dramatically changed and improved our understanding of this proteiform disease. Briefly, the idea is that there are two different types of mass-forming intrahepatic cholangiocarcinoma (MF-iCCA). This concept was originally proposed by a Japanese liver pathologist, Professor Okuda, back in 1977, stating that MF-ICCAs located around the porta hepatis resembled extrahepatic bile duct carcinoma, and those located within the liver parenchyma resembled hepatocellular carcinoma (HCC) [1]. Since then, extensive investigation has been done, revealing that these two subtypes came with significant differences, in terms of cellular origin, clinical features, macroscopic or microscopic appearance, background or environmental factors, and abnormalities in genetic and protein expression [2-9]. In the current volume of European Radiology, Kim et al report subtyping of MFiCCA solely based on contrast-enhanced MR imaging findings, successfully stratifying the prognosis of these two subgroups [10]. In this commentary, the background of this concept is briefly explained, along with the importance of Kim et al's report.

This comment refers to the article available at https://doi.org/10.1007/ s00330-018-5898-9.

Kengo Yoshimitsu

kengo@ fukuoka-u.ac.jp

1 Department of Radiology, Faculty of Medicine, Fukuoka University, 7-45-1 Nanakuma, Jonan-ku, Fukuoka 814-0180, Japan
To a better understanding, we need to go deep into the biological features of this tumor, enlightening the differences between the subtypes of cholangiocarcinoma:

1. Biliary anatomy and subtypes of MF-iCCA

Intrahepatic bile ducts are usually defined as a secondorder or more peripheral branches of the biliary system. MF-iCCAs are divided into those arising from large bile ducts close to the hilum (segmental to subsegmental ducts), which are called perihilar MF-iCCA, and those arising from peripheral ducts (septal or interlobular ducts), which are called peripheral MF-iCCA [1-3].

Another tumor arising from the most peripheral bile ducts, namely ductules, is separately called cholangiolocellular carcinoma (CoCC), which is not regarded as MF-iCCA, but defined as a subtype of combined carcinomas according to 2010 WHO classification. However, it could nevertheless be considered as an extreme form of the same spectrum of MF-iCCA [1-3].

2. Macroscopic and histologic differences between subtypes

Perihilar MF-iCCA is characterized by periductal spread, in addition to an intraparenchymal mass formation or not and consists of columnar tumor cells, presenting as a tubular adenocarcinoma with abundant mucin production. Intense desmoplastic fibrous stroma is also observed throughout the tumor, frequently associated with perineural invasion and poor prognosis [1-3]. Peripheral MF- 
iCCA is mainly an intraparenchymal mass-forming tumor and consists of low columnar or cuboidal tumor cells, presenting as a small ductal adenocarcinoma, with occasionally fused ducts, thick tumor plates, or compact growth pattern. It mimics CoCC or HCC [1-3]. Desmoplastic fibrous stroma is less prominent as compared with perihilar type and is confined to the center of the tumor. Mucin production is observed less frequently than in perihilar type. At the periphery of the tumor, portal tracts are occasionally preserved and surrounded by tumor cells, which is a frequently observed feature in CoCC as well.

3. Differences in background disease and precancerous conditions

Background disease for perihilar MF-iCCA includes hepatolithiasis, primary sclerosing cholangitis, parasitic disease, congenital biliary dilatation, and malfusion of the pancreatico-biliary duct, all of which are related to chronic inflammatory processes of the biliary system. In these conditions, biliary intraepithelial neoplasia (BilIN) is often observed, which would suggest an association between perihilar MF-iCCA and dysplasia-carcinoma sequence or a multi-step cholangiocarcinogenesis [1-3]. Conversely, for peripheral MF-iCCA, viral hepatitis (types B and C) infection and cirrhosis of any kind are the background diseases, which are chronic inflammatory processes of the liver parenchyma. Noteworthy, these are the background diseases for CoCC and HCC as well. Interestingly, p62-positive hyaline body, often seen in $\mathrm{HCC}$, can be found in peripheral MF-iCCA [4], and bile duct carcinoma can be experimentally induced from hepatocytes [5]. Altogether, these findings suggest a strong association between peripheral MF-iCCA carcinogenesis and chronic liver damage [1-3].

4. Differences in genetic abnormalities and expression of abnormal protein

$\mathrm{K}$-ras gene mutation is associated with infiltrative growth pattern, perineural invasion, poor prognosis, and perihilar-type MF-iCCA [2]. S100P protein is correlated to the histological grades of BilIN and is found in more than $90 \%$ of perihilar-type MF-iCCA, but rarely in peripheral-type MF-iCCA [6]. CD10, which is expressed on the stromal fibroblasts and facilitates cancer cell growth and invasion, is more frequently observed in perihilar MF-iCCA than in peripheral MF-iCCA [7]. Interestingly, the abovementioned three phenomena are also frequently seen in pancreatic ductal adenocarcinoma, suggesting the commonality between perihilar MF-iCCA and pancreas cancer.
On the other hand, NCAM (CD56) and N-cadherin, both cell adhesion-related molecules, are frequently observed in peripheral MF-iCCA, but extremely rarely in perihilar MF-iCCA [8].

5. Different stem/progenitor cells of the biliary system

There are two different types of stem/progenitor cells $(\mathrm{SPgC})$ in the biliary system. One is in the peribiliary glands located in the extrahepatic and large intrahepatic bile ducts (segmental and subsegmental) and the other in the bile ductules or canal of Hering, connecting the small bile ducts and bile canaliculi. The location of the former corresponds to where perihilar MF-iCCA occurs and is directly influenced by intraductal changes, especially chronic inflammation. Conversely, the location of the latter is outside of the confinement of the connective peri-portal tissue, which means that epithelial cells of the bile ductules, including SPgC, directly face the surrounding hepatocytes. Therefore, they are exposed to environmental changes within the liver parenchyma like chronic inflammation. Recent investigation has shown that $\mathrm{SPgC}$ in the bile ductules can develop into both hepatocytes and cholangiocytes $[2,9]$.

Thus, considering all of the abovementioned issues, $\mathrm{SPgC}$ in the peribiliary glands of large bile ducts and those at the bile ductules may be strongly associated with the carcinogenesis of perihilar and peripheral MFiCCAs, respectively. Kim et al discuss in their report that the two subtypes, namely ductal and parenchymal types, may well correspond to conventional perihilar and peripheral MF-iCCAs, respectively, as shown above. Of course, even if these two sets of subtypes do not match completely, Kim et al successfully stratified the prognosis of the two subgroups based on their classifying method. It is clinically relevant, as this information is obtained preoperatively and non-invasively.

In the future, it will be interesting and useful to clarify which classification system stratifies better the prognosis of the patients, or which combination of imaging features may best stratify the patients for the prognosis, using, for instance, a radiomics approach. The study by Kim et al is a very important step towards a better understanding and management of cholangiocarcinoma, as quite different tumors probably hide behind this single name.

Funding The author sates that this work has not received any funding.

\section{Compliance with ethical standards}

Guarantor The scientific guarantor of this publication is Kengo Yoshimitsu. 
Conflict of interest The author of this manuscript declares no relationships with any companies whose products or services may be related to the subject matter of the article.

Statistics and biometry No complex statistical methods are necessary for this paper.

Informed consent Written informed consent was not required for this study because this is not a study but an Editorial Comment.

Ethical approval Institutional Review Board approval was not required because this is not a study but an Editorial Comment.

\section{Methodology}

- Observational

\section{References}

1. Aishima S, Kuroda Y, Nishihara Y et al (2007) Proposal of progression model for intrahepatic cholangiocarcinoma: clinicopathologic differences between hilar type and peripheral type. Am J Surg Pathol 31:1059-1067

2. Nakanuma Y, Sato Y, Harada K et al (2010) Pathological classification of intrahepatic cholangiocarcinoma based on a new concept. World J Hepatol 2:419-427

3. Komuta M, Govaere O, Vandecaveye V et al (2012) Histological diversity in cholangiocellular carcinoma reflects the different cholangiocyte phenotypes. Hepatology 55:1876-1888
4. Aishima S, Fujita N, Mano Y et al (2010) p62+ Hyaline inclusions in intrahepatic cholangiocarcinoma associated with viral hepatitis or alcoholic liver disease. Am J Clin Pathol 134:457-465

5. Fan B, Malato Y, Calvisi DF et al (2012) Cholangiocarcinomas can originate from hepatocytes in mice. J Clin lnvest 122:2911-2915

6. Aishima S, Fujita N, Mano Y et al (2011) Different roles of SIOOP overexpression in intrahepatic cholangiocarcinoma : carcinogenesis of perihilar type and aggressive behavior of peripheral type. Am J Surg Pathol 35:590-598

7. Nishihara Y, Aishima S, Hayashi A et al (2009) CDIO+ fibroblasts are more involved in the progression of hilar/extrahepatic cholangiocarcinoma than of peripheral intrahepatic cholangiocarcinoma. Histopathology 55:423-431

8. Yu TH, Yuan RH, Chen YL et al (2011) Viral hepatitis is associated with intrahepatic cholangiocarcinoma with cholangiolar differentiation and N-cadherin expression. Mod Pathol 24:810-819

9. Carpino G, Cardinale V, Onori P et al (2012) Biliary tree stem/ progenitor cells in glands of extrahepatic and intrahepatic bile ducts: an anatomical in situ study yielding evidence of maturational lineages. J Anat 220:186-199

10. Rhee H, Kim MJ, Park YN, An C (2019) A proposal of imaging classification of intrahepatic mass-forming cholangiocarcinoma into ductal and parenchymal types: clinicopathologic significance. Eur Radiol. https://doi.org/10.1007/ s00330-018-5898-9

Publisher's note Springer Nature remains neutral with regard to jurisdictional claims in published maps and institutional affiliations. 